\title{
Fitogeografía del Valle de Tehuacán-Cuicatlán
}

\author{
José Luis Villaseñor, ${ }^{1-2}$ Patricia Dávila $^{2}$ y Fernando Chiang ${ }^{1}$
}

\begin{abstract}
RESUMEN. La provincia Florística del Valle de Tehuacán- Cuicatlán abarca una superficie aproximada de $10,000 \mathrm{~km}^{2}$ y en ella se encuentran alrededor de 630 géneros y 1,400 especies de plantas vasculares (Dávila, 1983). Su clima es predominantemente semiárido y su flora destaca por su elevado porcentaje de endemismo. En este trabajo se analiza la flora genérica desde el punto de vista de sus afinidades geográfico-climáticas y de sus relaciones florísticas con otras regiones geográficas. Las afinidades tropicales de esta flora genérica, en particular las neotropicales, predominan sobre todas las demás, siguiéndole en importancia el elemento característicamente mexicano. Las relaciones florísticas con otras regiones geográficas son significativas, lo que indica una fuerte correspondencia con la mayoría de ellas, sobre todo con las que tienen vegetación xerófila, revelándose también una mayor semejanza florística con localidades sudamericanas que con el Oeste de Norteamérica. Todo esto roloca al valle en una posicion fitogeográfica "estratégica", donde mejor se observa la participación del componente típicamente tropical y de aquel propio de las zonas áridas más septentrionales, lo que explica la gran riqueza florística encontrada en tan pequeña área.
\end{abstract}

ABSTRACT. The Tehuacán-Cuicatlán Valley Floristic Povince covers an area of approximately $10,000 \mathrm{~km}^{2}$, with ca. 630 genera and 1,400 species of vascular plants (Davila, 1983). Its climate is predominantly semiarid and its endemic flora is notably rich. The generic flora is analyzed from the viewpoint of its geographic and climatic affinities, as well as its floristic relationships with other geographical regions. The tropical affinities, particularly the neotropical ones, of this generic flora, prevail over all others: the characteristically Mexican element being second in importance. The floristic relationships with other geographical regions are significant, pointing to a strong correspondence with most of them, especially with those with a xerophytic vegetation, showing also a tronger floristic resemblance to some South American regions

1 Departamento de Botánica, Instituto de Biología, Universidad Nacional Autónoma de México, Apdo. Postal 70-233, Delegación Coyoacán, 04510 México, D. F.

2 Dirección actual: Rancho Santa Ana Botanic Garden, 1500 N. College Avenue, Claremont, California 91711, USA. 
than with western North America. All of this places the Tehuacán-Cuicatlán Valley in a strategic phytogeographic position, where the commingling of the typically tropical component with that which is characteristic of more northern arid zones can be best observed, which accounts for the great floristic richness found in such a small area.

La provincia florística del Valle de Tehuacán-Cuicatlán forma parte de la region xerofítica mexicana (Rzedowski, 1978) y se localiza en la parte sureste del estado de Puebla y noroeste del estado de Oaxaca, entre los 17 39' y 18 53' de latitud norte y los $9655^{\prime}$ y 97 44' de longitud oeste (fig. 1).

Desde el punto de vista fisiográfico, forma parte de la provincia denominada Mixteca-Oaxaqueña (Tamayo, 1962) y abarca varios valles, entre los que destacan los de Cuicatlán, Huajuapan, Tehuacán, Tepelmeme y Zapotitlán, que a su vez forman parte de la Cuenca Alta del Río Papaloapan y en menor proporción de la Cuenca Alta del Río Balsas. Sus límites orográficos principales son al este y noreste la Sierra Madre Oriental, aqui llamada Sierra de Zongolica y la Sierra de Juárez al sur. Los valles que conforman la provincia están limitados por una serie de serranías que en conjunto se conoce como Sierra Mixteca, la cual forma parte de la Sierra Madre Oriental. Su clima es de tipo semiárido, con temperatura alta, régimen de lluvias de verano con canícula y con poca a extremosa oscilación de temperatura. Este clima se debe fundamentalmente al efecto de sombra de lluvia que producen las sierras de Juárez y Zongolica.

La vegetación de esta provincia ha sido estudiada por Miranda (1947, 1948), Ramírez (1948); y recientemente por Ledezma (1979); Cruz-Cisneros y Rzedowski (1980); Zavala (1980, 1982) y Jaramillo y González Medrano(1983). Los tipos de vegetación, siguiendo la clasificación de Rzedowski (1978) son básicamente el bosque tropical caducifolio, el bosque espinoso, el bosque de encinos, el pastizal y el matorral xerófilo; este último es el más ampliamente distribuido y presenta una gran variación, dependiendo del elemento fisonómicamente dominante; entre los ejemplos más evidentes de esta variación se encuentran las tetecheras de Neobuxbaumia tetetzo (Weber) Backeberg, los izotales de Yucca periculosa Baker, los cardonales de Stenocereus weberi (Coulter) Buxbaum, los quiotillales de Escontria chiotilla (Weber) Rose y diversos tipos de matorrales espinosos, inermes o parvifolios- esclerófilos. Bordeando los ríos de la región y en algunos arroyos intermitentes es común ver bosques de galería.

El espectro altitudinal de estas comunidades oscila entre los 545 y los 2,458 m, aunque la media altitudinal de esta provincia son los 1,500 $\mathrm{msnm}$.

Desde el punto de vista geológico, el Valle de Tehuacán-Cuicatlán presenta afloramientos de diversa naturaleza y edad, que hacen de la región una serie de mosaicos con caractcrísticas y manifestaciones propias (López, 1981). Este autor se refiere a esta zona como parte de la provincia geológica de Tlaxiaco. La parte centro-norte de Tchuacán presenta afloramientos del Cretácico. Dentro de la región centro- sureste del valle, desde Tehuacán hasta la zona de Teotitlán del Camino, se presentan afloramientos del Precámbrico, así como elementos del Jurásico Inferior Marinn. Por último, la 


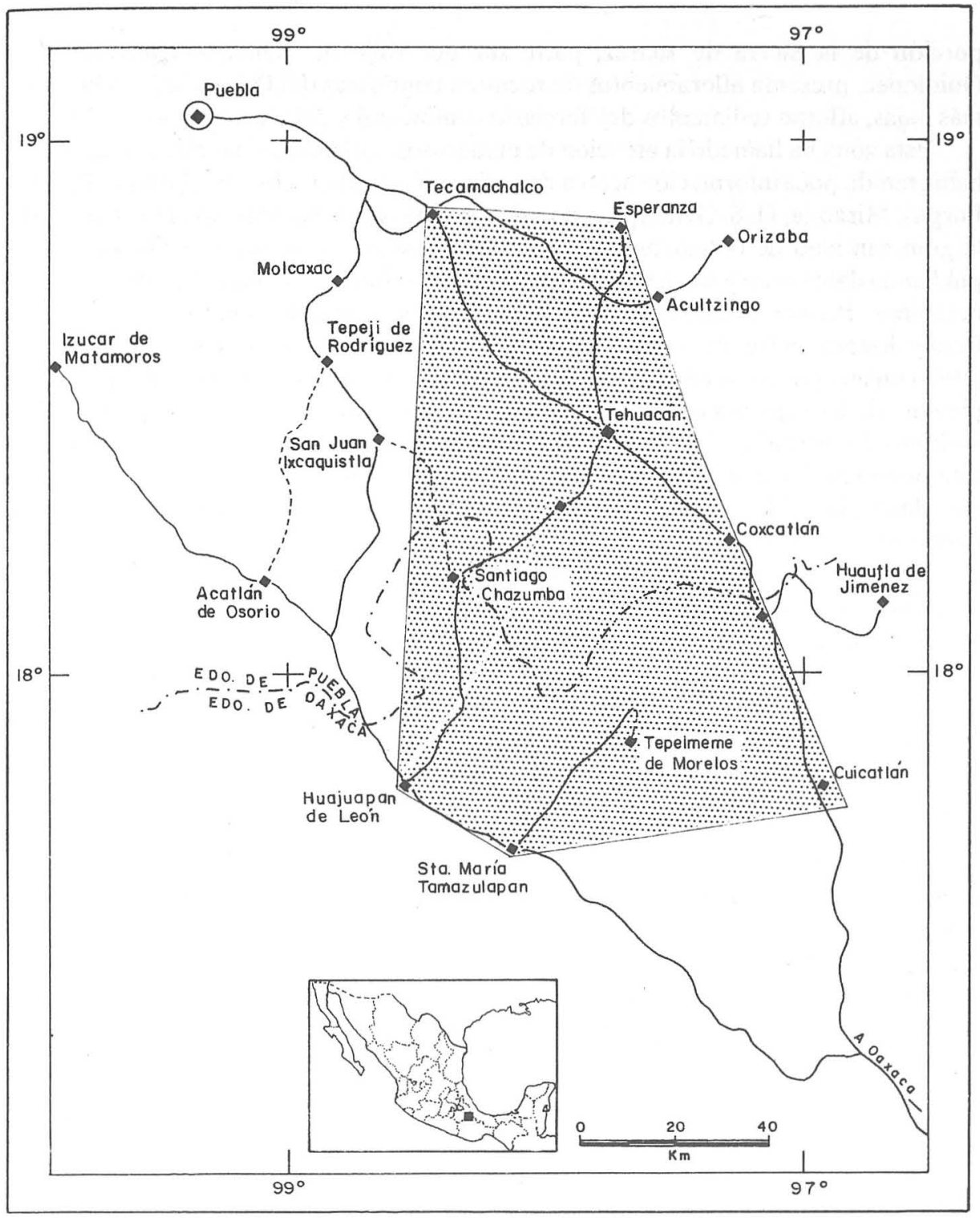

Valle de Tehuacán - Cuicallán

Figura 1. Mapa de la Provincia Florística de Tehuacán-Cuicatlán. 
porción de la Sierra de Juárez, parte sur del Valle de Tehuacán-Cuicatlán hasta Quiotepec, presenta afloramientos de rocas metamórficas del Paleozoico; en las partes más bajas, afloran sedimentos del Terciario continental y del Cuaternario.

Esta zona ha llamado la atención de numerosos botánicos; sin embargo, siempre se había tenido poca información acerca de su flora. Galeotti, Liebmann, Pringle, Conzatti, Purpus, Miranda, H. S. Gentry, Rzedowski y muchas personas más pueden citarse entre la gran cantidad de botánicos que han puesto interés en los recursos florísticos, han publicado datos acerca de su flora y vegetación o han hecho recolecciones de ejemplares botánicos. Pero no es sino hasta 1965 cuando Smith da una idea muy generalizada de la flora y destaca, entre otras cosas, su alto número de endemismos. Delgadillo y Zander (1984) en un estudio de musgos del Valle de Tehuacán, por el contrario, encuentran que ninguna de las especies de briófitas estudiadas por ellos es endémica a la zona. En el Instituto de Biología, UNAM, se viene realizando, desde 1979, un estudio florístico de esta provincia; hasta la fecha (Dávila, 1983) se han identificado 633 géneros de plantas vasculares, incluidos en 123 familias y comprendiendo alrededor de 1,460 especies (Tabla 1).

En la presente contribución se analizan los patrones de distribución de las gimnospermas y angiospermas que forman parte de la flora de esta provincia, así como sus relaciones con otras regiones geográficas de México y América.

\section{PATRONES DE Distribución de las FAMILIAS y GÉNEROS PRESENTES EN EL VALLE DE TEHUACÁN-CUICATLÁN}

Hemsley (1879-1888) y Sharp (1953) resumen los patrones de distribución de las familias, principalmente de angiospermas, presentes en la flora mexicana. Adaptando sus datos a las 118 familias de angiospermas y gimnospermas encontradas en esta provincia (Tabla 2), se tiene que el componente más significativo es el tropical; le siguen en importancia las familias con distribución en regiones templadas y cosmopolita. Once familias encontradas en la región bajo estudio son endémicas de América y ocho de estas familias endémicas se encuentran preferentemente en las partes áridas y semiáridas del continente.

Raven y Axelrod (1974) y recientemente Gentry (1982) subrayan la importancia fitogeográfica de la tectónica de placas en la distribución de las plantas. Dichos autores consideran a la flora tropical americana formada por dos elementos principales, aquellos derivados en el norte (Laurasia) y aquellos de afinidad meridional (Gondwana). De estos dos elementos, el componente preponderante es el de afinidad meridional, aunque en algunos casos el dominante desde el punto de vista fisonómico es de afinidad boreal. Con base en los datos presentados por estos autores, podemos decir que 44 familias de angiospermas del Valle de Tehuacán-Cuicatlán manifiestan una afinidad meridional, en tanto que 41 presentan una afinidad boreal, quedando 29 familias con una asignacion 
TABLA 1 Datos Generales sobre la flora de la Provincia Florística de Tehuacán-Cuicatlán

Familias reconocidas de Plantas Vasculares

Géneros

Especies
123

633

ca, 1,460

\section{FAMILIAS GENEROS ESPECIES}
Pteridofitas
5
12
ca. 33
Gimnospermas
4
5
12
Angiospermas
114
616
ca. 1,415

123

633

ca. 1,460 
TABLA 2 Distribución de las familias de Embryophyta Siphonogama presentes en la Provincia Florística de Tehuacán-Cuicatlán

FAMILIAS \%

Tropical y Subtropical

Templada

Cosmopolita y Subcosmopolita

Principalmente en América

Endémica de México y porciones adyacentes
1

10

8.5

de E. U. A.

25

21.2

23

19.5

50.0

9

(1)


difícil, debido a sus amplios patrones de distribución o a un origen probablemente más reciente. Aunque aparentemente existe una participación equitativa a nivel de familia entre ambas afinidades, no es sino al estudiar la proporción genérica con la que participan cuando uno puede apreciar la relevancia del elemento meridional en la flora de esta provincia.

En la tabla 3 se indican las familias que cuentan con más de 10 géneros en la región de estudio, así como su distribución. Igualmente se indica su afinidad, boreal o meridional (según Raven y Axelrod, 1974 y Gentry, 1982), es decir, con posibles centros de origen en Laurasia o Gondwana respectivamente.

Por otra parte, el análisis de la distribución de los 621 géneros de angiospermas y gimnospermas también refleja la dominancia del componente tropical en esta flora. Este componente tropical, que incluye más del $58 \%$ de todos los géneros, proviene de dos fuentes principales; la primera y mas importante la constitutyen aquellos elementos distribuidos en la region intertropical americana. Muchos de estos géneros con distribución neotropical, como en el caso de Brickellia (Robinson,1917); Dahlia (Sorensen, 1969); Galinsoga (Canne, 1977); Haplopappus (Urbatsch, 1978); Tithonia (LaDuke, 1982);Zinnia (Torres, 1963), etcétera. La segunda parte está representada por aquellos géneros que presentan una distribución pantropical, aunque resulta difícil incluir en este rubro géneros tales como Desmanthus, Leucaena, Phaseolus, etc.; éstos se encuentran representados por una o más especies en las regiones tropicales del mundo y podrían considerarse como de distribución pantropical, pero la mayoría de sus especies son mexicanas (Sousa, comunicación personal) y por ello debería considerarse que su distribución pantropical es secundaria.

Después del elemento tropical, el componente más importante es el de los géneros restringidos a territorio mexicano y regiones adyacentes. Este elemento autóctono tiene tres componentes: el de los géneros endémicos a la provincia de Tehuacán-Cuicatlán, el de los géneros endémicos a México y el de aquellos géneros distribuidos en México y regiones adyacentes de Estados Unidos y Guatemala. El elemento autóctono incluye 80 géneros, que representan el 13\% de la flora genérica.

Es importante indicar que a nivel específico el componente endémico al valle es todavía más importante; las estimaciones actuales corroboran las afirmaciones de otros autores (e g. Smith, 1965) acerca del alto grado de endemismo en la provincia. Cerca del $30 \%$ de las especies conocidas para esta zona son endémicas, lo cual es un alto porcentaje para una área tan pequeña.

Los elementos tropical y autóctono constituyen aproximadamente el $72 \%$ del total de la flora génerica; el resto de la flora de este valle está constituido por géneros de afinidad boreal y el elemento de distribución cosmopolita. Gran parte del elemento con afinidad boreal está asociado a los bosques de encinos y pastizales encontrados en la región. Rzedowski (1978) ha comentado la estrecha afinidad boreal de la flora de los pastizales propios de climas áridos y semiáridos. Además de estos elementos, está el de 
TABLA 3 Familias con mayor número de géneros en la Provincia Florística de Tehuacán-Cuicatlán

FAMILIA

Compositae
Gramineae
Leguminosae
Cactaceae
Euphorbiaceae

Scrophulariaceae

Malvaceae

Rubiaceae

Solanaceae

Acanthaceáe

Malpighiaceae

Liliaceae

Rosaceae

Boraginaceae

Convolvulaceae

Labiatae

Verbenaceac

\section{DISTRIBUCION}

Cosmopolita

Cosmopolita

Cosmopolita

Cosmopolita

Amplia, pero principal-

mente en los trópicos

Cosmopolita

Amplia, pero principal-

mete en los trópicos

Tropical

Tropical y Subtropical

Tropical y Subtropical

America, principalmente

Amplia, pero principal-

mente en los trópicos

Cosmopolita

Subcosmopolita, ausente

en las regiones mas frías

Tropical y Subtropical

Cosmopolita

Tropical y Subtropical
GENEROS

82

51

48

21

20

16

15

15

14

13

13

12

12

11

11

11

11

376

Otras

2
48
21

Meridional

No asignada

Meridional

Meridional

Meridional

Boreal

No Asignada

Meridional

Meridional

Meridional

Meridional

Boreal

Boreal

Boreal

Meridional

Boreal

No Asignada

Total

1 La afinidad expresa el probable centro de origen para la familia, según los datos de Raven y Axclrod (1974) y Gentry (1982). Boreal indica un probable origen en Laurasia, Meridional en Gondwana y No asignada cuando sus patrones de distribución son complejos y es difícil por el momento definir con seguridad su probable centro de origen. 
los géneros que presentan una distribución disyunta, principalmente entre las zonas cálido-secas de Norte y Sudamérica. Dentro de este componente con distribución disyunta podemos mencionar como ejemplos de extracción norteamericana a los géneros Celtis, Cercidium, Cryptantha, Dalea, Flourensia, Grindelia, Hoffmanseggia, Hymenoxys, Nama, Schkuhria, Sanvitalia, Tiquilia, Zaluzania, etc., y como ejemplos de extracción sudamericana a los géneros Aloysia, Castela, Condalia, Gochnatia, Grabowskia, Maytenus, Nicotiana, Prosopis, etc. También hay algunos otros géneros que relacionan a la flora de esta provincia con otras regiones más apartadas, por ejemplo Asia (Leibnitzia, Tiquilia, etc.) y Africa (Celosia, Cleome, etc.).

En la tabla 4 se resumen los patrones de distribución que presentan los géneros que conforman la flora del Valle de Tehuacán- Cuicatlán. Quedarían nada más por mencionar aquellos géneros que no manifiestan una gran especialización ecológica o climática y que por lo tanto son ampliamente encontrados a casi todo lo largo del continente americano, y aquellos que de manera consciente o inconsciente (e.g. Sonchus, Taraxacum, Lactuca, etc.), han sido introducidos a la provincia y han adquirido carta de naturalización.

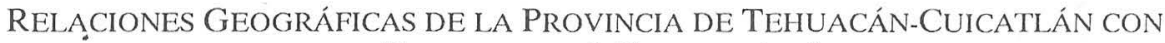 OTRAS REGIONES DE MÉXICO Y AMÉRICA}

Rzedowski (1973) discutió las relaciones florísticas de las zonas áridas de México, pero señaló la imposibilidad de estudiar en detalle esta provincia florística por carecer, en aquel tiempo, de un listado florístico. Es nuestra intención, una vez mostradas las principales afinidades de la flora de esta zona, retomar el enfoque fitogeográfico de este autor y complementar su aportación con la información florística ahora disponible. Para ello se analizan las relaciones de este valle con otras regiones de México y de América, algunas de las cuales cuentan, al menos parcialmente, con zonas de vegetación xerófila.

Los datos de esta provincia florística se compararon con los de otras 23 áreas geográficas, las cuales, siguiendo un arreglo latitudinal de norte a sur son:

California (Munz, 1973)

Arizona (Lehr, 1978)

Nuevo México (Wooton y Standley, 1915)

Texas (Correll y Johnston, 1970)

Baja California (Wiggins, 1980)

Desierto Sonorense (Shreve y Wiggins, 1964)

Bolsón de Cuatro Ciénegas, Coahuila (Pinkava, 1979-1981)

Chamela, Jalisco (Lott, 1985)

Valle de San Luis Potosí (Calderón, 1960)

Cuenca del Río Istórax, Querétaro (Zamudio, 1984)

Valle del Mezquital, Hidalgo (González, 1968)

Cuenca del Río Balsas (Soto, inédito) 
TABLA 4 Distribución de los géneros de Embryophyta Siphonogama presentes en la Provincia Florística de Tehuacán-Cuicatlán

GENEROS \%

Neotropicales

233

37.5

Pantropicales

132

21.3

Cosmopolitas

61

9.8

Endémicos al Valle đe Tehuacán-Cuicatlán

3

0.5

Endémicos a México

39

6.3

Distribuidos en México, Guatemala y sur de E.U.A.

38

6.1

Boreales

54

8.7

Con distribución disyunta

26

4.2

Ampliamente distribuidos en América

23

Naturalizados

12

1.9 


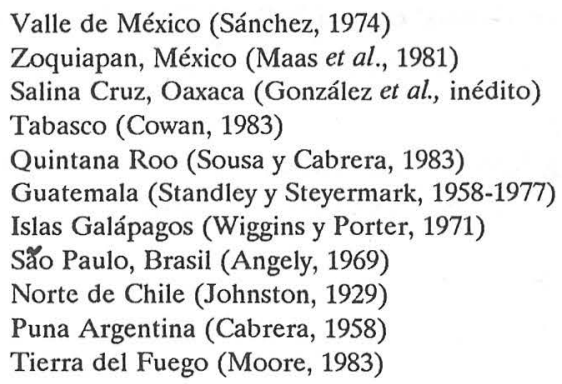

Se obtuvieron coeficientes de similitud según la fórmula de Preston, misma utilizada por Rzedowski (1973). Los resultados (tabla 5) indican que hay un alto grado de relación entre esta provincia y la mayoría de las regiones de México consideradas, tengan o no vegetación xerófila. Las zonas eminentemente xerófilas, como Cuatro Ciénegas, Valle del Mezquital o Desierto Sonorense, tienen coeficientes de similitud no muy diferentes a los de zonas característicamente tropicales, como Chamela, Cuenca del Río Balsas, Salina Cruz o Tabasco.

El bajo valor del coeficiente con respecto a California se debe a la diferencia climático-florística del suroeste de Estados Unidos, ya comentada por algunos autores (e.g. Rzedowski, 1973). Es interesante notar que hay valores casi del mismo orden o superiores con respecto a localidades sudamericanas, lo que indica una vez más la mayor relación florística con la flora meridional que con la flora boreal, de la cual derivó la vegetación que caracteriza al suroeste de Estados Unidos.

El resto del suroeste de Estados Unidos al parecer participa fuertemente del mismo componente florístico que muchas regiones mexicanas, pues los valores relativamente altos obtenidos en este análisis así parecen indicarlo. La relevancia de esta provincia se hace más evidente cuando se le compara con otras areas mexicanas. Los resultados obtenidos con localidades de muy distinta condición climática parecen indicar que esta región juega un importante papel en los patrones de distribución de muchos componentes de la flora de México.

Es un hecho que los elementos tropicales están bien representados en las zonas xerófilas del noroeste de México, específicamente en el Desierto Sonorense (Rzedowski, 1973). La pequeña zona árida de la Cuenca del Río Balsas así como el Valle de Tehuacán-Cuicatlán también manifiestan un alto porcentaje de elementos tropicales, no propiamente xerófitos en su flora. Además, es en esta última región donde muchos de los elementos propios de las zonas áridas de Norteamérica, por ejemplo los géneros Aphanostephus, Chrysactinia, Zaluzania, etcétera, tienen un límite de distribución meridional.

Lo anterior coloca a esta región como una zona de ecotonía entre la vegetación xerófila de la parte norte y la vegetación tropical de la parte sur del territorio nacional; este hecho es uno de los responsables de su gran riqueza florística a pesar de su pequeña 
TABLA 5 Géneros en común y coeficientes de similitud entre el Valle de Tehuacán-Cuicatlán y otras localidades de México y América

\section{GENEROS GENEROS COEFICIENTE DE TOTALES EN COMUN SIMILITUD (\%) ${ }^{1}$}

\begin{tabular}{|c|c|c|c|}
\hline Valle de Tehuacán-Cuicatlán & 621 & & \\
\hline California & 1001 & 230 & 39.8 \\
\hline Arizona & 919 & 326 & 57.5 \\
\hline Nuevo México & 826 & 264 & 47.8 \\
\hline Texas & 173 & 406 & 68.6 \\
\hline Baja California & 861 & 348 & 62.3 \\
\hline Desierto Sonorense & 806 & 351 & 64.1 \\
\hline \multicolumn{4}{|l|}{ Bolsón de Cuatro Ciénegas } \\
\hline Coahuila & 427 & 274 & 70.5 \\
\hline Chamela, Jalisco & 420 & 234 & 61.0 \\
\hline Valle de San Luis Potosí & 405 & 283 & 75.9 \\
\hline \multicolumn{4}{|l|}{ Cuenca del Río Estórax, } \\
\hline Querétaro & 383 & 305 & 85.5 \\
\hline Valle del Mezquital, Hidalgo & 280 & 244 & 89.9 \\
\hline Cuenca del Río Balsas & 679 & 427 & 83.0 \\
\hline Valle de México & 499 & 290 & 66.8 \\
\hline Zoquiapan, México & 111 & 62 & 56.0 \\
\hline Salina Cruz, Oaxaca & 291 & 199 & 70.8 \\
\hline Tabasco & 751 & 353 & 66.0 \\
\hline Quintana Roo & 632 & 275 & 55.3 \\
\hline Guatemala & 1715 & 482 & 79.0 \\
\hline Islas Galápagos & 309 & 156 & 52.6 \\
\hline São Paulo, Brasil & 1413 & 279 & 46.3 \\
\hline Norte de Chile & 198 & 64 & 32.7 \\
\hline Puna Argentina & 263 & 93 & 36.3 \\
\hline Tierra del Fuego & 260 & 48 & 18.9 \\
\hline
\end{tabular}

1. Coeficiente de Similitud utilizado: Preston (1962, En Rzedowski, 1973). 
superficie, varias decenas de veces más pequeña que, por ejemplo, California o Texas, pues en ella confluyen plantas tanto del norte como del sur.

\section{CONCLUSIONES}

Muchos factores intervienen en la gran riqueza florística del Valle de TehuacánCuicatlán. Su posición geográfica, su clima, su fisiografía, etc., hacen de esta provincia florística uno de los sitios de mayor interés botánico. En este valle encuentran su límite meridional algunas comunidades vegetales propias de climas áridos y semiáridos de Norteamérica, como son los pastizales y los matorrales gipsófilos. Tal vez es en esta zona donde mejor se puede observar la penetración del elemento tropical, propio de zonas cálido-húmedas, hacia las zonas áridas y semiáridas.

Todos estos factores contribuyen a que este valle tenga importantes relaciones florísticas tanto con las zonas áridas del norte de México como con las zonas tropicales del sur, las cuales se prolongan incluso hasta algunas regiones sudamericanas.

El gran número de endemismos de este valle indica un fuerte aislamiento, tal vez desde un tiempo muy remoto (Rzedowski, 1978), pero sus altas afinidades florísticas también indican una importante relación con muchas otras provincias florísticas de México.

Finalmente, la preponderancia del elemento tropical en la flora xerófila de México es un hecho confirmado por otros autores; dicho predominio también se observa en esta provincia florística y concuerda con la importancia que en segundo término presenta el elemento restringido al territorio mexicano y zonas vecinas.

AgradecimIENTOS. Los Dres. Claudio Delgadillo, Rodolfo Dirzo y Jerzy Rzedowski tuvieron la amabilidad de leer el manuscrito y se les agradecen sus observaciones críticas.

\section{LITERATURA CITADA}

ANGELY, J. 1969. Flora analítica e fitogeográfica do Estado de São Paulo. Ed. Phyton. São Paulo. 2. CABRERA, A. L. 1958. La vegetación de la República Argentina VI. La vegetación de la Puna Argentina. Inagro 11(4):317-412.

CALDERÓN DE R., G. 1960. Notas sobre la flora y la vegetación del estado de San Luis Potosí VII. Vegetación del Valle de San Luis Potosí. Acta Ci. Potosina 4:5-118.

CANNE, J. M. 1977. A revision of the genus Galinsoga (Compositae: Heliantheae). Rhodora 79:319-389. CORRELL, D. S. y M. C. JOHNSTON. 1970 Manual of the vascular plants of Texas. Texas Research Foundation. Renner, Texas. 1881p. 
COWAN, C. P. 1983. Listados Florísticos de México I. Flora de Tabasco. Instituto de Biología, U.N.A.M. México. 123p.

CRUZ-CISNEROS, R. y J. RZEDOWSKI. 1980. Vegetación de la Cuenca del Río Tepelmeme, Alta Mixteca, estado de Oaxaca (México). Anales Esc. Nal. Ci. Biol. 22:19-84.

DÁVILA A., P. 1983. Flora genérica del Valle de Tehuacán-Cuicatlán. Tesis, Maestro en Ciencias. Facultad de Ciencias, UNAM, México. 694p.

DELGADILLO M., C. y R. H. ZANDER. 1984. The mosses of the Tehuacan Valley, Mexico, and notes on their distribution. Bryologist 87:319-322.

GENTRY, A. H. 1982. Neotropical floristic diversity: Phytogeographical connections between Central and South America, Pleistocene climatic fluctuations, or an accident of the Andean orogeny? Ann. missouri Bot. Gard. 69:557-593.

GONZÁLEZ Q., L. 1968. Tipos de vegetación del Valle del Mezquital, Hidalgo. Departamento de Prehistoria del Instituto Nacional de Antropología e Historia, México. 53p.

HEMSLEY, W. B. 1879-1888. Botany. En: Godwin, F. D. y O. Salvin. Biologia Centrali Americana. R. H. Porter, London. V. 4.

JARAMILLO L., V. y F. GONZÁLEZ MEDRANO. 1983. Análisis de la vegetación arbórea de la provincia florística de Tehuacán- Cuicatlán. Bol. Soc. Bot. México 45:49-64.

JOHNSTON, I. M. 1929. Papers on the flora of Northern Chile I. The coastal flora of the Departments of Chanaral and Taltal. Contr. Gray Herb. 85:1-138.

LA DUKE, J. C. 1982. Revision of Tithonia. Rhodora 84:453-522.

LEDEZMA M., A. R. 1979. Tipos de vegetacion y algunas caracteristicas ecológicas en que se desarrolla en los municipios de Caltepec y Zapotitlan Salinas, Puebla, Mexico. Tesis, Universidad Autónoma de Nuevo León, México.

LEHR, J. H. 1978. A catalogue of the flora of Arizona. Desert Botanical Gard. Phoenix, Arizona. 203p.

LÓPEZ R., E. 1981. Geología de México. Publ. particular autorizada tomo III. Mexico. 446p.

LOTT, E. J. 1985. Listados Morísticos de México. III. La estación de biología Chamela, Jalisco. Instituto de Biología, UNAM.

MASS, J., R. PATRÓN, A. SUÁREZ, S. BLANCO, G. CEBALLOS, C. GALINDO y A. PESCADOR. 1981. Ecología de la estación experimental Zoquiapan (Descripción general, vegetación y fauna). Colección Cuadernos Universitarios Serie Agronomía No. 2. Universidad Autónoma Chapingo, México. $115 \mathrm{p}$.

MIRANDA, F. 1947. Estudios sobre la vegetación de México V. Rasgos de la vegetación en la Cuenca del Río de las Balsas. Revisia Soc. Mex. Hist. Nat. 8(1-4):95-114.

MIRANDA, F. 1948. Datos sobre la vegetación en la Cuenca Alta del Río Papaloapan. Analles Inst. Biol. Univ. Nac. México 19(2):333-364.

MOORE, D. M. 1983. Flora of Tierra del Fuego. Anthony Nelson. England. 396 p.

MUNZ, P. A. 1973. A California flora with supplement. University of California Press. Berkeley and Los Angeles. $1681+22 \mathrm{p}$.

PINKAVA, D. J. 1979-1981. Vegetation and flora of the Bolsón of Cuatro Ciénegas Region, Coahuila, Mexico. Bol. Soc. Bot. México 38:35-44; 39:107-127; 41:127-151.

RAMÍREZ C., D. 1948. Anotaciones generales sobre la vegetación acuática ruderable y arvense de Cuicatlán y sus alrededores. Anales Inst. Biol. Univ. Nac. México 19(2):427-440.

RAVEN, P. H. y D. AXELROD. 1974. Angiosperm biogeography and past continental movements. Ann. Missouri BoL. Gard. 61(3):539-673.

ROBINSON, B. L. 1917. A monograph of the genus Brickellia. Mem. Gray Herb. 1:1-151.

RZEDOWSKI, J. 1973. Geographical relationships of the flora of Mexican dry regions. En: Graham, A. (Edr.). Vegetation and vegetational history of Northern Latin America. Elsevier, Amsterdam. pp. 61-72.

RZEDOWSKI, J. 1978. Vegetación de México. Limusa. México. 432p. 


\section{FITOGEOGRAFIA DEL VALLE DE TEHUACAN-CUICATLAN}

SÁNCHEZ S., O. 1974. La flora del Valle de México. 2a. ed. Herrero Hnos., México. 519p.

SHARP, A. J. 1953. Notes on the flora of Mexico; world distribution of the woody dicotyledonous families and the origin of the modern vegetation. J. Ecol. 41:374-380.

SHREVE, F. y I. L. WIGGINGS. 1964. Vegetation and flora of the Sonoran Desert. Stanford University Press, Stanford. $2 \mathrm{~V}$.

SMITH, C. E. 1965. Flora, Tehuacan Valley. Fieldiana, Bot. 31(4):107-143.

SORENSEN, P. D. 1969. Revision of the genus Dahlia (Compositae- Heliantheae: Coreopsidineae). Rhodora 71:309-365, 367-416.

SOUSA S., M. y E. F. CABRERA C. 1983. Listados florísticos de México II. Flora de Quintana Roo. Instituto de Biología, UNAM, México. 100p.

STANDLEY, P. C. y J. STEYERMARK. 1958-1977. Flora of Guatemala. Fieldiana, Bot. 24. Parts I-XIII.

TAMAYO, J. L. 1962. Geografía general de México. 2a. ed. Instituto Mexicano de Investigación Económica, México. $4 \mathrm{~V}$.

TORRES, A. M. 1963. Taxonomy of Zinnia. Brittonia 15:1-25.

URBATSCH, L. E. 1978. The Chihuahuan Desert species of Ericameriac (Compositae: Astereae). Sida 7:298-303.

WIGGINS, I. L. 1980. Flora of Baja California. Stanford University Press, Stanford. 1025p.

WOOTON, E. O. y P. C. STANDELY. 1915. Flora of New Mexico. Contr. U. S. Natl. Herb. 19:9-794.

ZAMUDIO R., S. 1984. La vegetación de la Cuenca del Río Eslórax, en el estado de Querétaro y sus relaciones fitogeográficas. Tesis. Facultad de Ciencias, UNAM. México. 275p.

ZAVALA H., J. A. 1980. Estudios ecológicos en el Valle semiárido de Zapotitlán de las Salinas, Puebla. Clasificación de la vegetación. Tesis. Facultad de Ciencias, U.N.A.M. México. 159p.

ZAVALA H., J. A. 1982. Estudios ecológicos en el Valle de Zapotitlán, Puebla. I. Clasificación numérica de la vegetación basada en atributos binarios de presencia de las especies. Biotica 7:99- 120. 